\title{
ON SOME FUNCTIONS OF LITTLEWOOD-PALEY AND ZYGMUND
}

BY E. M. STEIN

Communicated by P. R. Halmos, August 21, 1960

In a previous paper [2], we studied the $n$-dimensional form of the functions of Littlewood-Paley and Lusin. These were defined as follows. Let $f(x) \in L^{p}\left(E_{n}\right), E_{n}$ is Euclidean $n$-space, of variables $x, y, \cdots, x=\left(x_{1}, x_{2}, \cdots, x_{n}\right)$; let $U(x, t), t>0$, be the Poisson integral of $f$. Let

$$
g(x)=\left(\int_{0}^{\infty} t|\nabla U|^{2} d t\right)^{1 / 2} \text { and } S(x)=\left(\iint_{W(x)} t^{1-n}|\nabla U|^{2} d t d y\right)^{1 / 2} .
$$

Here

$$
|\nabla U|^{2}=\sum_{k=0}^{n}\left(\frac{\partial U}{\partial x_{k}}\right)^{2}, \quad x_{0}=t ;
$$

$W(x)$ is the cone $\{(y, t):|x-y|<\alpha t\}$. We proved

$$
B_{p}\|f\|_{p} \leqq\|g\| \leqq A_{p}\|f\|_{p}, \quad 1<p<\infty,
$$

with a similar result for $S$.

We wish now to consider a related function of Littlewood-Paley and Zygmund. We define its $n$-dimensional version as follows. Let $0<\lambda$, and set

$$
g_{\lambda}^{*}(x ; f)=g_{\lambda}^{*}(x)=\left(\int_{0}^{\infty} \int_{E_{n}} \frac{t^{\lambda+1}}{\left(|x-y|^{2}+t^{2}\right)^{(\lambda+n) / 2}}|\nabla U|^{2} d y d t\right)^{1 / 2} .
$$

We note first

$$
g(x) \leqq A S(x) \leqq B_{\lambda} g_{\lambda}^{*}(x) .
$$

The first part of this inequality is Lemma 9 of [2], and the second part is trivial. We note also that $g_{\lambda_{1}}^{*}(x) \leqq g_{\lambda_{2}}^{*}(x)$, if $\lambda_{2} \leqq \lambda_{1}$. We shall see that the behavior of $g_{\lambda}^{*}$ when $\lambda>n$ is similar to that of the simpler functions $g$ and $S$. Hence our primary concern will be with $g_{\lambda}^{*}$ when $0<\lambda \leqq n$. We outline the proof of the following theorem.

Theorem. Let $0<\lambda \leqq n$, and $2 n /(\lambda+n)<p<\infty$. Then

$$
\left\|g_{\lambda}^{*}\right\|_{p} \leqq A_{p, \lambda}\|f\|_{p}, \quad A_{p, \lambda} \text { independent of } f .
$$


Remarks. (i) For the one-dimensional periodic case see Zygmund [5]; for the nonperiodic case see Waterman [4]. The proofs given there are based on complex methods, which of course are unavailable in higher dimensions.

(ii) The result stated here is essentially the best possible: there exists an $f \in L^{1}$ so that $g_{n}^{*}(x)=\infty$, almost everywhere; also if $0<\lambda<n$, and $p<2 n /(\lambda+n)$, there exists an $f \in L^{p}$, so that $g_{\lambda}^{*}(x)=\infty$, a.e.

The proof follows a series of steps.

Lemma 1. If $0<\lambda, 2 \leqq p<\infty$, then

In fact

$$
\left\|g_{\lambda}^{*}\right\| \leqq A_{p, \lambda}\|f\|_{p} .
$$

$$
\left\|g_{\lambda}^{*}\right\|_{p}^{2}=\sup \int \frac{t^{\lambda}}{\left(|x-y|^{2}+t^{2}\right)^{(n+\lambda) / 2}}|\nabla U|^{2} \phi(x) d x d y d t,
$$

the sup is taken over all $\phi \geqq 0,\|\phi\|_{r} \leqq 1$, where $r$ is the index conjugate to $p / 2$. However,

$$
\begin{aligned}
\sup _{t>0} \int \frac{t^{\lambda}}{\left(|x-y|^{2}+t^{2}\right)^{(n+\lambda) / 2}} \phi(x) d x \\
\qquad A \sup _{t>0} t^{-n} \int_{|x| \leq t} \phi(y-x) d x=A M(\phi)(y) .
\end{aligned}
$$

Therefore by Fubini's theorem,

$$
\left\|g_{\lambda}^{*}\right\|_{p}^{2} \leqq A \int g^{2}(y) M(\phi)(y) d y \leqq A\|g\|_{p}^{2}\|M \phi\|_{r} \leqq B\|f\|_{p}^{2} .
$$

Here we have used inequality (1), and a well-known inequality concerning the "maximal function" $M(\phi)$.

Lemma 2. Let $n<\lambda$, then the operation $f \rightarrow g_{\lambda}^{*}$ is of weak type $(1,1)$.

The proof of this lemma is based on the same ideas as the analogous Lemma 12 of [2], for the functions $g$ and $S$.

LEMMA 3. Let $n<\lambda$, then

$$
\left\|g_{\lambda}^{*}\right\|_{p} \leqq A_{p, \lambda}\|f\|_{p}, \quad 1<p<\infty .
$$

This follows from a combination of Lemma 1 (when $n<\lambda$ ), Lemma 2 and the Marcinkiewicz interpolation theorem.

We can now prove the theorem. Let $\phi(x, y, t)=\phi=\left(\phi_{0}, \phi_{1}, \cdots, \phi_{n}\right)$ be a vector-valued function so that $\int_{0}^{\infty} \int_{E_{n}}|\phi(x, y, t)|^{2} d y d t \leqq 1$, all $x$, but let $\phi$ be arbitrary otherwise. Let 


$$
T_{\lambda}(f)(x)=\int_{0}^{\infty} \int_{E_{n}} \frac{t^{(\lambda+1) / 2}}{\left(|x-y|^{2}+t^{2}\right)^{(n+\lambda) / 4}}\left(\sum_{k=0}^{n} \frac{\partial U}{\partial x_{k}} \cdot \phi_{k}\right) d y d t .
$$

Then $T_{\lambda}$ is a family of linear operators depending analytically on $\lambda$, and satisfying

$$
\begin{array}{ll}
\left\|T_{\lambda}(f)\right\|_{p} \leqq A_{p, \lambda_{0}}\|f\|_{p}, & 2 \leqq p<\infty, R(\lambda)=\lambda_{0}>0, \\
\left\|T_{\lambda}(f)\right\|_{p} \leqq A_{p, \lambda_{1}}\|f\|_{p}, & 1<p<\infty, R(\lambda)=\lambda_{1}>n .
\end{array}
$$

The bounds $A_{p, \lambda_{0}}$ and $A_{p, \lambda_{1}}$ are independent of $\phi$. We may now apply the convexity theorem of [1] and interpolate between (2) and (3). The result is $\left\|T_{\lambda}(f)\right\|_{p} \leqq B_{p, \lambda}\|f\|_{p}$, if $2 n /(\lambda+n)<p<\infty . B_{p, \lambda}$ is independent of $\phi$. Taking the sup over $\phi$ proves the theorem.

We shall now remark briefly on the applications of the functions $g, S$, and $g_{\lambda}^{*}$. The function $g$ is basic in the Littlewood-Paley theory of Fourier series (see e.g. [7, Chapter 15]). The $n$-dimensional extension of these results is as yet unknown. The function $S$ is decisive in the behavior of harmonic functions near the boundary; the $n$-dimensional results have recently been obtained; see [3]. An application of the function $g_{\lambda}^{*}$ is one variable is given in [6]. In the following paper we shall apply the $n$-dimensional results to the characterization of certain classes of functions arising by "fractional integration."

\section{BiBLIOGRAPHY}

1. E. M. Stein, Interpolation of linear operators, Trans. Amer. Math. Soc. vol. 83 (1956) pp. 482-492.

2. - On the functions of Littlewood-Paley, Lusin, and Marcinkiewicz, Trans. Amer. Math. Soc. vol. 88 (1958) pp. 430-466.

3. - On harmonic functions in several variables. I and II, Notices Amer. Math. Soc. vol. 7 (1960) Abstracts 566-35 and 566-36.

4. D. Waterman, On functions analytic in a half-plane, Trans. Amer. Math. Soc. vol. 81 (1956) pp. 167-194.

5. A. Zygmund, On certain integrals, Trans. Amer. Math. Soc. vol. 55 (1944) pp. 170-204.

6. - On the Littlewood-Paley function $g^{*}(\theta)$, Proc. Nat. Acad. Sci. U.S.A. vol. 42 (1956) pp. 208-212.

7. - Trigonometric series, 2nd ed., Cambridge, Cambridge University Press, 1959.

\section{University of Chicago}

